\title{
Same-sign trilepton signal for stop quark in the presence of sneutrino dark matter
}

\author{
Dilip Kumar Ghosh* \\ Department of Theoretical Physics, Indian Association for the Cultivation of Science Jadavpur, \\ Kolkata 700 032, India \\ Katri Huitu ${ }^{\dagger}$ and Subhadeep Mondal ${ }^{\ddagger}$ \\ Department of Physics, and Helsinki Institute of Physics, P. O. Box 64, FI-00014 \\ University of Helsinki, Finland \\ Manimala Mitra ${ }^{\S}$ \\ Institute of Physics, Sachivalaya Marg, Bhubaneswar, Odisha 751005, India, \\ Homi Bhabha National Institute, Training School Complex, Anushakti Nagar, Mumbai 400085, India
}

(Received 29 November 2018; published 15 April 2019)

\begin{abstract}
We explore a minimal supersymmetric standard model scenario extended by one pair of gauge singlets per generation. In the model, light neutrino masses and their mixings are generated via the inverse seesaw mechanism. In such a scenario, a right-handed sneutrino can be the lightest supersymmetric particle and a cold dark matter (DM) candidate. If the Casas-Ibarra parametrization is imposed on the Dirac neutrino Yukawa coupling matrix $\left(Y_{\nu}\right)$ to fit the neutrino oscillation data, the resulting $Y_{\nu}$ is highly constrained from the lepton-flavor-violating decay constraints. The smallness of $Y_{\nu}$ requires the sneutrino DM to coannihilate with other sparticle(s) in order to satisfy the DM relic density constraint. We study sneutrino coannihilation with winos and observe that this sneutrino-wino compressed parameter space gives rise to a novel same-sign trilepton signal for the top squark, which is more effective than the conventional top squark search channels in the present framework. We show that the choice of neutrino mass hierarchy strongly affects the signal event rate, making it easier to probe the scenario with the inverted mass hierarchy.
\end{abstract}

DOI: 10.1103/PhysRevD.99.075014

\section{INTRODUCTION}

The existence of nonzero neutrino masses and mixings $[1,2]$ remains one of the greatest unsolved problems of physics and one of the strongest motivations for looking for physics beyond the standard model (BSM). Supersymmetry (SUSY) remains one of the frontrunners among various $\mathrm{BSM}$ candidates. However, the $R$-parity-conserving minimal supersymmetric standard model (MSSM) is unable to address the neutrino mass problem. The easiest way to overcome this shortcoming is to incorporate a seesaw mechanism into the framework, where the light neutrinos gain nonzero masses through small mixing with additional

\footnotetext{
*tpdkg@iacs.res.in

katri.huitu@ @elsinki.fi

subhadeep.mondal@helsinki.fi

§manimala@iopb.res.in
}

Published by the American Physical Society under the terms of the Creative Commons Attribution 4.0 International license. Further distribution of this work must maintain attribution to the author(s) and the published article's title, journal citation, and DOI. Funded by SCOAP. gauge singlet fields. The canonical seesaw mechanism [3-7] adds three generations of gauge singlet superfields to the MSSM particle content. The light neutrino mass scale leads to very small Dirac neutrino Yukawa couplings $\left(Y_{\nu}\right)$ in the presence of $\mathrm{TeV}$-scale right-handed neutrinos. The smallness of $Y_{\nu}$ makes it difficult to observe any effects from the neutrino sector at the LHC. Lepton-number- and/or leptonflavor-violating (LFV) decays, on the other hand, can constrain these parameters further with increasing sensitivities. A more phenomenologically interesting option is provided by the inverse seesaw mechanism [8-10], where $Y_{\nu}$ can in principle be as large as $\mathcal{O}(0.1)$ owing to the presence of a small lepton-number-violating parameter in the theory. Such large Yukawa parameters and sub-TeV right-handed neutrino masses can be constrained from collider as well as low-energy experiments [11-13].

The presence of a right-handed ( $\mathrm{RH})$ neutrino superfield in a SUSY theory provides us with the exciting possibility of obtaining a RH sneutrino as the lightest SUSY particle (LSP), which can also be a good cold dark matter (DM) candidate [14-25]. The left-handed sneutrino LSP option is strongly disfavored from DM direct detection constraints 
due to its gauge coupling with the $Z$ boson [26,27]. Therefore, in order to be considered as a DM candidate, a sub-TeV sneutrino LSP has to be RH. This RH sneutrino arises from a singlet superfield and thus only couples to other particles via the Yukawa couplings. The DM experimental data can be another probe to test the neutrino sector parameters in models augmented with a gauge singlet superfield. The only pair annihilation process of any significance involves Higgs bosons in the $s$ channel, and unless the sneutrino mass is close to the scalar resonance region, the annihilation is not enough to produce the correct relic density [17-19]. Thus, a sneutrino DM mass around $\frac{m_{h}}{2}$, where $m_{h}$ indicates the $125 \mathrm{GeV}$ Higgs boson mass, has been favored by DM constraints. However, this region is now under severe scrutiny with the improvements in DM direct detection constraints.

In the absence of the Higgs resonance region, it is quite difficult to produce a large enough annihilation cross section for the RH sneutrinos in such scenarios. This forces us to look at coannihilation options. With proper coannihilation, a sneutrino can be a viable DM candidate throughout the mass range $[100 \mathrm{GeV}-1 \mathrm{TeV}]$. However, direct search constraints on the other sparticles indirectly put constraints on the sneutrino masses in such cases. Attractive options involve winos or Higgsinos as the nextto-lightest SUSY particle (NLSP) since they always present the possibility of coannihilation with more than one particle simultaneously. Thus, the DM constraints naturally lead to a compressed electroweak sector involving the LSP sneutrino and multiple neutralino-chargino states as the NLSP. As a result of this compression, the decay products of the NLSP neutralino-chargino are expected to be soft, resulting in weaker exclusion limits. This also means that such a scenario will be hard to detect from the direct production of NLSP pairs. One can, however, produce them in a cascade and look for a multilepton channel with a small SM background to distinguish the signal.

One such possibility can arise from top squark production and its subsequent decay into the LSP sneutrino via the neutralino-charginos. The physics of the top squark is of utmost importance in MSSM-like theories that require adding a substantial correction to the tree-level Higgs boson mass in order to increase it up to $125 \mathrm{GeV}$. This correction appears mostly from the top squark loop, making the top squark mass and mixing parameters relevant for SUSY searches at the LHC. The existing constraints on the top squark mass can extend up to $1 \mathrm{TeV}$ depending on its various decay modes [28-34]. At the same time, this exclusion limit can be relaxed in the presence of a compressed electroweak sector, as we do here. It turns out that the existing search channels are not sensitive enough to probe top squark masses effectively under such circumstances owing to the poor signal rates. Hence, we construct a same-sign trilepton signal region, which is nearly background free and thus-despite a poor event rate—can be used to probe $\mathrm{TeV}$-order top squark masses at relatively low luminosity. We also show that the choice of normal hierarchy (NH) or inverted hierarchy (IH) in the light neutrino masses is reflected in the final event rate, which highlights a nice correlation between the neutrino and top squark sectors and a unique characteristic of such neutrino mass models.

The paper is organised as follows. In Sec. II, we briefly give an overview of the model and describe how neutrino oscillation data has been fit. In Sec. III we discuss the phenomenological constraints on the model arising from LFV decays, DM searches, and collider experiments. In Sec. IV we discuss the canonical top squark search strategies at the LHC and propose for the same a novel same-sign trilepton signal region, which is more suitable to probe the present scenario. Then we go on to define a few benchmark points representative of the parameter space of our interest and perform a detailed collider analysis to present our results in the context of the $13 \mathrm{TeV}$ LHC. We show the exclusion limits on the top squark mass derived from this study at moderate and high luminosities at the LHC in the case of a null result. In this context we also present the limits that can be expected at a high-energy (27 TeV) hadron machine. In Sec. V we summarize our results and conclude.

\section{MODEL}

The supersymmetric inverse seesaw model (SISM) contains the SM gauge singlet superfields $\hat{N}$ and $\hat{S}$ with lepton numbers -1 and +1 , respectively. The superpotential with these extra superfields is

$$
W=W_{\mathrm{MSSM}}+Y_{\nu} \hat{L} \cdot H_{u} \hat{N}+M \hat{N} \hat{S}+\mu_{S} \hat{S} . \hat{S} .
$$

In the above we consider three generations of $\hat{N}_{i}$ and $\hat{S}_{i}$ (i $=1,2,3)$ and $\mu_{S}$ violates lepton number by two units $(\Delta L=2)$. The soft supersymmetry-breaking Lagrangian for this model is

$$
\begin{aligned}
\mathcal{L}_{\text {soft }}= & \mathcal{L}_{\mathrm{MSSM}}-\left[m_{N}^{2} \tilde{N} \tilde{N}+m_{S}^{2} \tilde{S} \tilde{S}\right] \\
& -\left[A_{\nu} \tilde{L} H_{u} \tilde{N}+B_{1} \tilde{N} \tilde{S}+B_{2} \tilde{S} \tilde{S}+\text { H.c. }\right] .
\end{aligned}
$$

The $9 \times 9$ neutrino mass matrix in the basis $(\nu, N, S)$ has the following form:

$$
\mathcal{M}_{\nu}=\left(\begin{array}{ccc}
0 & M_{D} & 0 \\
M_{D}^{T} & 0 & M \\
0 & M^{T} & \mu_{S}
\end{array}\right),
$$

where $M_{D}, M$, and $\mu$ are $3 \times 3$ matrices and $M_{D}=Y_{\nu} v_{u}$, where $v_{u}$ is the vacuum expectation value (VEV) of $H_{u}$. For the parameter $\left\|\mu_{S}\right\| \ll\|M\|$, the light neutrino mass matrix becomes 


$$
M_{\nu} \sim M_{D} M^{T-1} \mu_{S} M^{-1} M_{D}^{T}
$$

The matrix $\mu_{S}$ can be small ${ }^{1}$ enough to explain the eV neutrino mass constraint. In passing, we would like to add a few comments on the choice of our superpotential. Since we want the lightest right-handed sneutrino state to be a stable LSP and hence a DM candidate, we choose to work within an $R$-parity-conserving framework. This choice prevents us from writing terms that violate lepton number by odd unit(s), e.g., $\hat{N} \hat{N} \hat{N}, \hat{N} \hat{N} \hat{S}, \hat{S} \hat{S} \hat{S}$, and $\hat{N} \hat{S} \hat{S}$. We do not allow the scalar components of $\hat{N}$ and $\hat{S}$ to obtain VEVs for the same reason. However, one can in principle allow additional $\Delta L=2$ terms, i.e., nonzero $\hat{L} \hat{S}$ and $\hat{N} \hat{N}$ terms in the superpotential. However, the presence of the $\hat{N} \hat{N}$ term does not affect the inverse seesaw structure at the tree level as the rank of $\mathcal{M}_{\nu}$ remains the same. On the other hand, the $\hat{L} \hat{S}$ term will modify Eq. (2.4) unless the coupling $Y_{S}$ in $Y_{S} \hat{L} . \hat{H}_{u} \hat{S}$ is very small, $Y_{S} \lesssim 10^{-12}$. One can impose additional symmetry $[36,37]$ in the superpotential to forbid such terms. We have chosen to work within a minimal setup [38-41] that can address the neutrino oscillation data via the inverse seesaw mechanism.

The light neutrino mass matrix is diagonalized by the Pontecorvo-Maki-Nakagawa-Sakata (PMNS) mixing matrix $U_{\nu}$ as follows:

$$
U_{\nu}^{T} M_{\nu} U_{\nu}^{*}=M_{\nu}^{d}
$$

We consider the matrices $M$ and $\mu_{S}$ to be diagonal. One can follow the Casas-Ibarra parametrization [42] to construct the matrix $R$,

$$
R=M_{\nu}^{d-1 / 2} U_{\nu}^{T} M_{D} M^{T-1} \mu_{S}^{1 / 2},
$$

where $R$ is a complex orthogonal matrix with $R R^{T}=I$. Throughout the paper we consider the best-fit values of the oscillation parameters [2]. The Dirac mass matrix $M_{D}$ can be fixed in terms of the light neutrino masses, PMNS mixing matrix, and $R$ as

$$
M_{D}=U_{\nu}^{*} \sqrt{M^{d}}{ }_{\nu} R \mu_{S}^{-1 / 2} M .
$$

In our subsequent discussion, we consider the simplest scenario with $R=\mathbb{I}$. The heavy neutrino masses are proportional to $M \pm \mu_{S}$. After diagonalization, this gives rise to the quasidegenerate sterile neutrino states with mass splitting $\delta M \sim \mu_{S}$.

\footnotetext{
${ }^{1}$ Note that $\mu_{S}$ breaks lepton number by two units. Hence, in the limit $\mu_{S} \rightarrow 0$ the symmetry of the theory is enhanced. From the naturalness argument, $\mu_{S}$ is preferred to be small. Moreover, a small $\mu_{S}$ is absolutely essential to incorporate the inverse seesaw mechanism. Moreover, a large $\mu_{S}$ will cause the $\Delta L=2$ processes like neutrinoless double beta decay to increase rapidly [35].
}

Below, we discuss a number of experimental constraints arising from the lepton-flavor-violating searches, dark matter searches, and collider constraints.

\section{EXPERIMENTAL CONSTRAINTS}

In this section, we discuss different experimental constraints on the heavy neutrino and sneutrino parameters in addition to the neutrino oscillation data. The heavy neutrinos in the inverse seesaw mechanism can give a large contribution in the loop-mediated LFV conversion processes $\ell_{i} \rightarrow \ell_{j} \gamma, \ell_{i} \rightarrow \ell_{j} \ell_{j} \ell_{j}$, and $\ell_{i} \rightarrow \ell_{j}$ in nuclei [38-41,43,44]. Additionally, for much lower masses (up to a few $\mathrm{GeV}$ ) the sterile neutrinos can be further constrained from different LFV meson decays $M \rightarrow M^{\prime} l_{i} l_{j}$, fixed-target experiments, the peak searches in $\pi \rightarrow e \nu$ and $K \rightarrow e \nu$, and $\beta$ decay [45]. Active-sterile neutrino mixing up to $\theta^{2} \sim 10^{-11}$ can be probed in the future FCC-ee [46] and SHiP experiments [47], which is relevant for the few$\mathrm{GeV}$ mass range of the $\mathrm{RH}$ neutrino. The SISM is augmented with small lepton number violation owing to the smallness of $\mu_{S}$. Therefore, the LNV processes, such as $0 \nu 2 \beta, K \rightarrow \pi l^{ \pm} l^{ \pm}$will be suppressed in this model. We consider the heavy neutrinos in the $[100 \mathrm{GeV}-1 \mathrm{TeV}]$ range that can be most optimally tested through the flavor violation and collider searches. In the SISM, $\ell_{i} \rightarrow \ell_{j} \gamma$ and $\ell_{i} \rightarrow \ell_{j}$ conversion in nuclei are further enhanced due to sneutrino-chargino- and slepton-neutralino-mediated loop contributions. We furthermore assume that the sneutrino is the LSP and obtain the constraints from the dark matter relic density.

\section{A. LFV constraints}

As an artifact of fitting the neutrino oscillation data as described in the previous section, the resulting Dirac neutrino mass matrix $M_{D}$ becomes off-diagonal which can potentially enhance LFV decays like $\ell_{i} \rightarrow \ell_{j} \gamma$. We keep the Yukawa matrix for the charged lepton diagonal. As a result, the LFV decays are generated at one loop through contributions from lepton-neutrino- $W$ boson, lepton-slepton-neutralino, and lepton-sneutrino-chargino loops. Both nonsupersymmetric and supersymmetric loop contributions have been studied in detail $[38,41,48]$. Depending on the choices of the neutrino sector parameters, namely, $M_{D}, M, \mu_{S}$, and the chargino and sneutrino masses, these LFV decay rates can be significant. The experimental limits on such LFV decay branching ratios [49-55] can thus effectively restrict the model parameter space. The existing constraints are more stringent for LFV decays of muons than of taus owing to the better detection efficiency of the former. Hence, stringent restrictions on the $Y_{\nu}$ parameters are to be expected when an inverted hierarchy of light neutrino masses is considered owing to the resultant large $Y_{\nu}$ corresponding to the first generation. In order to study the impact of these constraints on 

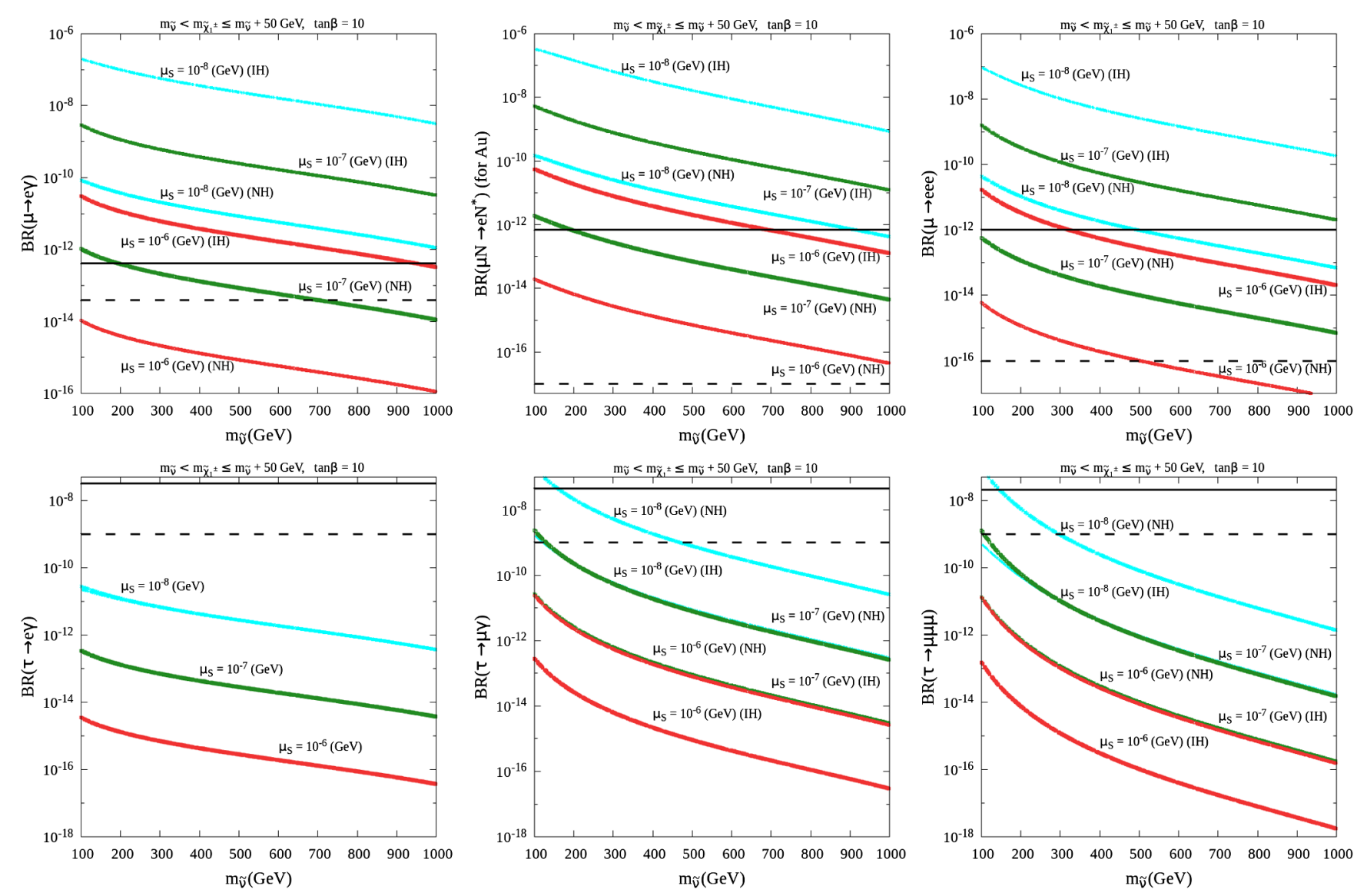

FIG. 1. Top panel: The predicted branching ratio of $\mu \rightarrow e \gamma, \mu-e$ conversion in nuclei and $\mu \rightarrow e e e$ vs the sneutrino mass. Lower panel: The branching ratios for $\tau \rightarrow e \gamma, \tau \rightarrow \mu \gamma$, and $\tau \rightarrow \mu \mu \mu$. The horizontal solid black lines correspond to the present experimental bounds on $\operatorname{BR}(\mu \rightarrow e \gamma) \leq 4.2 \times 10^{-13}, \operatorname{BR}\left(\mu N \rightarrow e N^{*}\right) \leq 10^{-12}, \operatorname{BR}(\mu \rightarrow e e e) \leq 10^{-12}, \operatorname{BR}(\tau \rightarrow e \gamma) \leq 3.3 \times 10^{-8}$, $\operatorname{BR}(\tau \rightarrow \mu \gamma) \leq 4.4 \times 10^{-8}$, and $\operatorname{BR}(\tau \rightarrow \mu \mu \mu) \leq 2.1 \times 10^{-8}$ [49-55]. The corresponding future sensitivities are shown by the horizontal dotted black lines [49-55].

the LSP mass and its couplings, we perform a scan where $M$ is varied within the range $[100 \mathrm{GeV}-1 \mathrm{TeV}]$. The wino mass parameter $M_{2}$ is adjusted in such a way that $m_{\tilde{\nu}_{1,2}}<$ $m_{\tilde{\chi}_{1}^{ \pm}} \leq m_{\tilde{\nu}_{1,2}}+50 \mathrm{GeV}$ to facilitate coannihilation with the LSP, which is discussed in the subsequent subsection. The value of $\tan \beta$ is kept fixed at 10 and different $\mu_{S}$ values $\left(10^{-8}, 10^{-7}\right.$, and $\left.10^{-6} \mathrm{GeV}\right)$ are chosen with $Y_{\nu}$ fitted according to Eq. (2.7). The computation of particle masses, mixing, and their decay are performed using SPHENO ${ }^{2}$ [56-58] after model implementation using SARAH [59-63]. For the LFV decay branching ratio calculations we use the results from Ref. [41] which include both the SUSY and non-SUSY contributions.

Figure 1 shows the variation of the relevant LFV decay branching ratios as a function of the LSP mass $m_{\tilde{\nu}_{1,2}}{ }^{3}$

\footnotetext{
${ }^{2}$ All of the masses are calculated with corrections up to one loop, apart from the Higgs mass which is computed up to the two-loop level.

${ }^{3}$ Heavy neutrino masses are the same as the sneutrino masses since they are both driven by the same parameter $M$.
}

The different colored lines correspond to different choices of $\mu_{S}$, while the black solid and dotted lines represent the present and future experimental sensitivities for the respective LFV processes. As expected, the constraints are most severe for the IH case in the $\mu \rightarrow e \gamma$ and $\mu N \rightarrow e N^{*}$ modes. In particular, for IH of light neutrino masses, the LNV parameter $\mu_{S}<10^{-6} \mathrm{GeV}$ is excluded throughout the entire LSP mass region up to $\tilde{m}_{\nu}=1000 \mathrm{GeV}$. The restriction is relatively much weaker from the LFV $\tau$ decays $\tau \rightarrow e \gamma$, $\tau \rightarrow \mu \gamma$, and $\tau \rightarrow \mu \mu \mu$. The experimental limits on these decay modes must be significantly improved to probe the parameter space in consideration. As expected, for the $\tau$ LFV decays, the normal mass hierarchy predicts a larger branching ratio than the inverted mass hierarchy because of the large third-generation Yukawa couplings. This could be easily tested in next-generation experiments. With the recent results of the neutrino oscillation experiment $\mathrm{NO} \nu \mathrm{A}$ disfavoring the $\mathrm{IH}$ in the neutrino sector $[64,65]$, the predictions for the $\mathrm{NH}$ are even more significant. For $\tau \rightarrow e \gamma$ the predicted branching ratio is smaller by more than $\mathcal{O}\left(10^{4}\right)$ than $\tau \rightarrow \mu \gamma$. In this case, the lines corresponding 
to $\mathrm{NH}$ and IH merge to result in one unique line for each $\mu_{S}$ value. $^{4}$ The restriction from $\tau \rightarrow e e e$ is the weakest, and hence we do not explicitly show it. As the future sensitivities show, the $\mu N \rightarrow e N^{*}$ conversion process would be the best-suited probe for the entire parameter space.

Apart from the neutrino sector parameters, the masses of the neutralino and charginos can also be constrained from these LFV decay computations. In order to get Fig. 1, we have only kept the wino mass close to the LSP as mentioned above. All other neutralino-chargino masses are kept above $2.0 \mathrm{TeV}$. Since the wino pair lies close to the LSP mass, some of the parameter region with a light enough sneutrino mass can be ruled out from the LHC data. However, LFV constraints on these masses depend on the choice of $\mu_{S}$. As shown in Fig. 1, a large $\mu_{S}$ results in weaker LFV constraints. We checked that for $\mu_{S} \sim 10^{-5} \mathrm{GeV}$, even for the more constraining inverted hierarchy scenario, sneutrino and chargino masses close to $200 \mathrm{GeV}$ are still allowed from the LFV decays. However, this parameter space will be ruled out from LHC direct search constraints on the gaugino masses. Note that LFV decay rates are not significant enough to constrain SUSY particle masses above $100 \mathrm{GeV}$ for $\mu_{S} \gtrsim 10^{-6} \mathrm{GeV}$ if we assume the $\mathrm{NH}$ of light neutrino masses.

\section{B. Dark matter constraints}

In the SISM, a light RH sneutrino cold DM candidate can be ideally fit in the Higgs resonance region [17-19], i.e., $m_{\tilde{\nu}_{1,2}}$ is required to lie in the vicinity of $m_{h} / 2$ (where $m_{h}$ is the SM-like Higgs mass) in order to ensure sufficient annihilation to satisfy the relic density constraints [66]. The $\mathrm{RH}$ sneutrino states are required to have sufficiently small mixing with the LH sneutrino states in order to avoid the direct detection cross section $\left(\sigma_{S I}\right)$ constraints. However, the most recent constraints on $\sigma_{S I}$ imposed by DM experiments like XENON1T and PANDA [67-69] have already excluded this parameter space. On the other hand, imposing the LFV constraints on the LSP mass and couplings renders the $Y_{\nu}$ parameters sufficiently small so that the resulting $\sigma_{S I}$ in the sub-TeV $\tilde{\nu}_{1,2}$ mass region lies orders of magnitude below the present experimental sensitivity [67-69]. However, such small $Y_{\nu}$ imply that even the Higgs resonance region cannot provide sufficient annihilation for the RH-sneutrino LSP to satisfy the relic density constraint. As a consequence, one has to look for the coannihilation of $\tilde{\nu}_{1,2}$ with other sparticles. Here we study the sneutrino coannihilation with wino-like charginos and neutralinos. The right-handed LSP sneutrino cannot interact directly with the wino components of the chargino-neutralino pair and can

\footnotetext{
${ }^{4}$ In the distributions of $\operatorname{BR}(\tau \rightarrow \mu \gamma)$ and $\operatorname{BR}(\tau \rightarrow \mu \mu \mu)$, the $\mathrm{IH}$ lines corresponding to the smaller $\mu_{S}$ overlap with the NH line corresponding to the subsequent $\mu_{S}$. The small numerical difference between the lines is indistinguishable on the logarithmic scales of the figures.
}

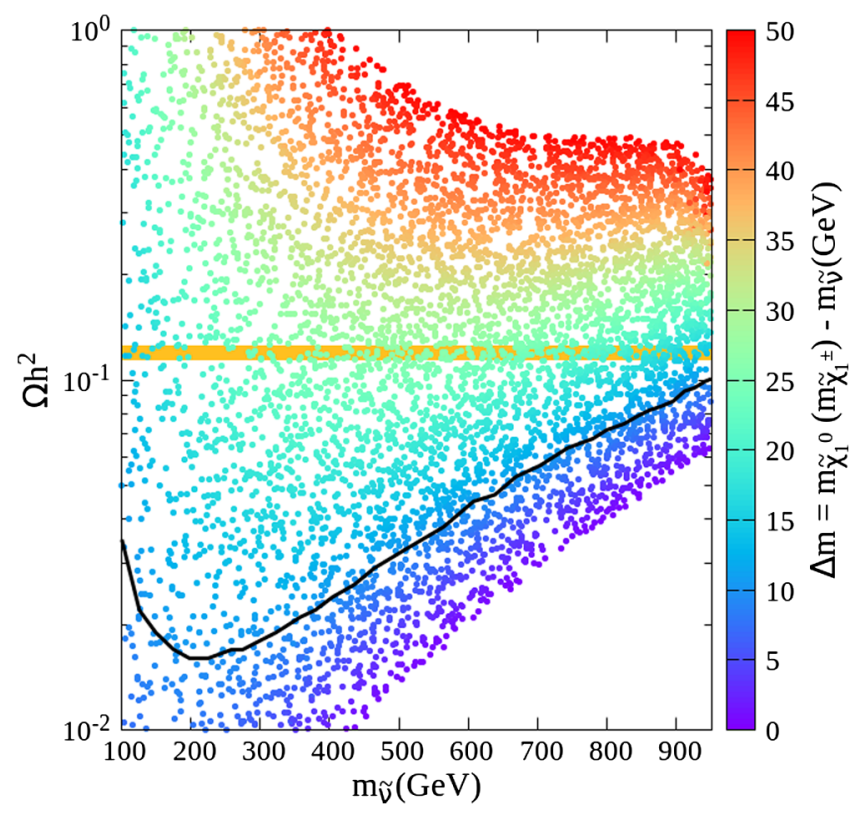

FIG. 2. The variation of the relic density is shown as a function of the LSP sneutrino mass. The color coding represents the LSPNLSP mass gap, $\Delta m$. The horizontal band represents the $2 \sigma$ allowed range of the relic density, $\Omega h^{2}=0.119 \pm 0.0027$ [66]. The black contour represents $\Delta m \sim 10 \mathrm{GeV}$.

only do so through their Higgsino component. However, we study the case where the NLSP chargino-neutralino pair is almost purely wino-like. Under this circumstance, the resultant relic density is entirely due to the coannihilation of the chargino-neutralino pair. The contribution of sneutrino annihilation to the relic density is negligibly small [70]. Hence, the LSP can be purely right-handed, which results in an elusively small direct detection cross section. We use MICROMEGAs [71] to calculate the relic density and direct detection cross section.

It is worth mentioning that a Higgsino NLSP can also be a good choice. Due to the presence of three nearly degenerate neutralino-chargino states that can coannihilate with the sneutrino, a Higgsino NLSP scenario is also capable of producing the correct relic density with appropriate choices of the Higgsino parameters. A bino LSP scenario, on the other hand, fails to meet the relic density criteria due to a lack of sufficient coannihilation channels.

Figure 2 shows the relic density $\left(\Omega h^{2}\right)$ distribution as a function of DM mass $m_{\tilde{\nu}_{1,2}}$ after taking into account the neutrino mass and LFV constraints. $\tan \beta$ is kept fixed at the moderate value $10^{5}$ and the $Y_{\nu}$ matrix is derived at each point according to Eq. (2.7). The choice of NH or IH does not have a significant impact on the distribution. However, we keep $\mu_{S}=10^{-5} \mathrm{GeV}$ in order to avoid the LFV

\footnotetext{
${ }^{5}$ The choice of $\tan \beta$ does not affect the sneutrino mass range or the LSP-NLSP mass gap required to produce the correct relic density.
} 
constraints. The parameter $M$ is varied in the range [1001000] $\mathrm{GeV}$ as before, and to ensure coannihilation the LSP-NLSP mass difference $\left(\Delta m=m_{\tilde{\chi}_{1}^{0}}\left(m_{\tilde{\chi}_{1}^{ \pm}}\right)-m_{\tilde{\nu}_{1,2}}\right)$ is kept within $50 \mathrm{GeV}$ by adjusting the wino mass parameter $M_{2}$. The $\Delta m$ variation is color coded according to the gradient bar on the right. The horizontal golden shaded region indicates the $2 \sigma$ allowed range of the relic density, $\Omega h^{2}=0.119 \pm 0.0027$ [66]. As evident from the figure, $15<\Delta m<30 \mathrm{GeV}$ is favored from $\Omega h^{2}$ considerations. Therefore, one can derive stringent constraints on the NLSP masses subjected to the sneutrino DM mass from the relic density requirement. The black contour in Fig. 2 shows the boundary below which $\Delta m<10 \mathrm{GeV}$. With such a small $\Delta m$, the decay products of the NLSP will practically be undetectable. However, in this work we are only concerned with the points lying above the relic-density-allowed band. Given the allowed $\Delta m$ corresponding to these points, the resulting NLSP decay products will be soft but detectable with varied efficiency. ${ }^{6}$

\section{Collider constraints}

Since the sneutrino DM requires coannihilation with a wino in the present scenario, the existing limits on the mass of the wino-like chargino and neutralino can further constrain the DM-allowed parameter region indirectly. The most stringent constraints from the LHC on the wino mass are derived from trilepton and/or dilepton final states resulting from $\tilde{\chi}_{2}^{0} \tilde{\chi}_{1}^{ \pm}$and $\tilde{\chi}_{1}^{ \pm} \tilde{\chi}_{1}^{\mp}$ production channels, respectively, with the assumption that $\tilde{\chi}_{2}^{0}$ and $\tilde{\chi}_{1}^{ \pm}$are wino-like and mass degenerate while the $\tilde{\chi}_{1}^{0}$ is the LSP and is bino-like [72-78]. However, in our scenario the wino-bino mass hierarchy is reversed and the sneutrino is the LSP, and thus the final decay products are kinematically quite different. The coannihilation requirement puts the wino $\tilde{\chi}_{1}^{0}-\tilde{\chi}_{1}^{ \pm}$pair close to the $\tilde{\nu}_{1,2}$ mass and, as a result, the leptons arising from the $\tilde{\chi}_{1}^{ \pm}$decay are quite soft. $\tilde{\chi}_{1}^{0}$ decay, on the other hand, is completely invisible. Besides, the $\tilde{\chi}_{2}^{0} \tilde{\chi}_{1}^{ \pm}$production cross section is much smaller here compared to that used by the experimental analyses because of the sizable mass gap between the two states. The $\tilde{\chi}_{1}^{ \pm} \tilde{\chi}_{1}^{0}$ production cross section is expected to be larger, but the monolepton signal has a huge background contribution arising from the single $W$ production channel at the LHC. Thus, the neutralino-chargino production channels result in much weaker limits on the relevant sparticle masses. Existing constraints from $\tilde{\chi}_{1}^{ \pm} \tilde{\chi}_{1}^{\mp}$ production can also restrict the SISM scenario. However, the resultant dilepton signal region requires relatively large lepton $p_{T}$ cuts that reduce the signal event rate drastically in our case. One can revisit the dilepton analyses of ATLAS and

\footnotetext{
${ }^{6}$ In our collider analysis, in order to attain maximal cut efficiency we consider $\Delta m \sim 25 \mathrm{GeV}$.
}

CMS in this regard with softer $p_{T}$ cuts on the leptons. Nevertheless, one has to use a large stransverse mass $\left(M_{T 2}\right)$ cut in order to get rid of the background arising from $W$-boson pair production. This cut discards most of the signal events from chargino pair production here because of the small chargino-sneutrino mass gap. Moreover, the absence of large transverse missing energy makes this channel less sensitive at the LHC. The existing exclusion limits are clearly not sensitive when the LSP-NLSP mass gap is $\sim 20-30 \mathrm{GeV}$, even with small $M_{T 2}$ cuts [72]. All of these factors combine to make the existing limits on the gaugino masses much more relaxed in our case and allow us to choose sufficiently light sneutrino DM masses. We have ensured that the existing constraints on the gaugino masses are properly taken into account by testing our parameter space against the LHC results via CHECKMATE [79,80].

\section{COLLIDER PHENOMENOLOGY}

Our discussion of the SISM scenario and the impact of the neutrino oscillation, LFV, and DM experimental constraints point towards a naturally compressed spectrum consisting of the LSP sneutrino and the NLSP wino-like neutralino-chargino pair. As discussed in the previous section, the canonical search strategy for the gauginos at the LHC may not be sensitive enough to probe this scenario or distinguish the signal from that expected from an MSSM compressed spectra. We observe that a clean and distinguishable signal region for our scenario can be obtained via the secondary production of the gauginos from a top squark cascade. Note that, although the choices of wino masses in this framework are subjected to the choice of the LSP mass, the bino-like neutralino $\left(\tilde{\chi}_{2}^{0}\right)$ and the colored sparticle masses can have any value as long as they are not excluded by the LHC data.

The top squark search at the LHC [29-34] mostly concentrates on the two top squark decay modes $\tilde{t} \rightarrow t \tilde{\chi}_{1}^{0}$ and $\tilde{t} \rightarrow \tilde{\chi}_{1}^{ \pm} b$, where $\tilde{\chi}_{1}^{0}$ and $\tilde{\chi}_{1}^{ \pm}$are bino- and wino-like, respectively. The various signal regions include zero-, one-, or two-lepton final states associated with $b$ jets and missing transverse energy $\left(E_{T}\right)$. The most stringent constraint on the top squark mass in the MSSM framework is derived by assuming either $\tilde{t} \rightarrow t \tilde{\chi}_{1}^{0}, \tilde{t} \rightarrow b \tilde{\chi}_{1}^{ \pm}\left(\tilde{\chi}_{1}^{ \pm} \rightarrow W \tilde{\chi}_{1}^{0}\right)$, or $\tilde{t} \rightarrow$ $b f f^{\prime} \tilde{\chi}_{1}^{0}$, where $f$ refers to the fermions. The existing bound on the top squark mass can extend up to $1 \mathrm{TeV}$ for a massless LSP $[32,33]$. Although similar final states can be obtained in our scenario these conventional decay branching ratios can be quite small, and at the same time the leptons originating from the NLSP decay $\tilde{\chi}_{1}^{ \pm} \rightarrow \ell \tilde{\nu}_{1,2}$ are expected to be soft and consequently result in a worse cut efficiency. Thus, the conventional search strategies for the lighter top squark may not be applicable to this scenario.

A very clean and unique final state can result from righthanded top squark pair production and subsequent decay of 
a top squark into a bino-like $\tilde{\chi}_{2}^{0}$ along with a top quark, while $\tilde{\chi}_{2}^{0}$ further decays to the wino-like chargino $\tilde{\chi}_{1}^{ \pm}$and a $W$ boson. The $\tilde{\chi}_{1}^{ \pm}$finally decays to a charged lepton and LSP sneutrino. Owing to the Majorana nature of the $\tilde{\chi}_{2}^{0}$, this cascade can arise at the LHC in a same-sign trilepton signal, which is almost background free. The full-decay chain that we consider here is, therefore, as follows:

$$
\begin{aligned}
& p p \rightarrow \tilde{t}_{1} \tilde{t}_{1}^{*} \rightarrow t \tilde{\chi}_{2}^{0} \bar{t} \tilde{\chi}_{2}^{0} \rightarrow b W^{+} \bar{b} W^{-} \tilde{\chi}_{1}^{ \pm} \tilde{\chi}_{1}^{ \pm} W^{\mp} W^{\mp}, \\
& \tilde{\chi}_{1}^{ \pm} \rightarrow \ell^{ \pm} \tilde{\nu}_{1,2} .
\end{aligned}
$$

The final state consists of $b \bar{b}+\mathrm{n}-$ jets $+\ell^{ \pm} \ell^{ \pm} \ell^{ \pm}+$ $E_{T}(\ell \equiv e, \mu)$, where two of the same-sign leptons originate from the two charginos, while the third originates from one of the $W$ bosons arising from top-quark decays. Note that a similar hierarchy in the top squark and gaugino masses can be obtained in the MSSM as well with a wino-like LSP. However, in that case, in order to result in a same-sign trilepton final state, three of the same-sign $W$ bosons in the cascade would have to decay leptonically. The final event rate is, therefore, expected to be smaller [28] due to the small $W$ leptonic decay branching ratio. However, in our present scenario, the NLSP $\tilde{\chi}_{1}^{ \pm}$can only decay leptonically $(e, \mu$, or $\tau)$ into the sneutrino and only one of the $W$ 's in the cascade is required to do the same. Hence, the event rate is expected to be much larger. On the other hand, the LSPNLSP compressed region $(\Delta m \simeq 25-30 \mathrm{GeV})$ results in softer leptons, which affects the cut efficiency and reduces the event rate somewhat, but the same-sign trilepton being a clean channel, proves to be much more effective in probing the SISM parameter space than the conventional signal regions.

\section{A. Sample benchmark points}

In Table I we present the relevant parameters, masses, branching ratios, and different experimental constraints corresponding to two sample benchmark points assuming $\mathrm{NH}$ and $\mathrm{IH}$ in each case.

For our choice of the sneutrino mass and lepton-numberviolating parameter $\mu_{S}$, the LFV branching ratios are well within the present experimental limits, and the IH case of $\mathrm{BP} 1$ is the most likely to be probed by $\operatorname{BR}(\mu \rightarrow e \gamma)$ in the near future. The DM direct detection cross sections are also rendered quite small. The choice of $\mathrm{NH}$ or IH is highlighted in the resulting $Y_{\nu}$ matrix. The larger third-generation $Y_{\nu}$ results in a larger decay branching ratio of the $\tilde{\chi}_{1}^{ \pm}$into $\tau \tilde{\nu}_{1,2}$ in the $\mathrm{NH}$ case. On the other hand, in the IH case, the larger first-generation $Y_{\nu}$ leads to a larger $\operatorname{BR}\left(\tilde{\chi}_{1}^{ \pm} \rightarrow e \tilde{\nu}_{1,2}\right)$. Naturally, one would expect larger leptonic event rates in the IH cases compared to the $\mathrm{NH}$ ones when the lighter chargino appears in the cascade. The $\tilde{t}_{1}$, being mostly righthanded, decays dominantly into a top quark and the binolike $\tilde{\chi}_{2}^{0}$. In the absence of the $\tilde{\chi}_{1}^{0} h$ mode, $\tilde{\chi}_{2}^{0}$ decays entirely into $\tilde{\chi}_{1}^{ \pm} W^{\mp}$. The mass difference between $\tilde{\chi}_{2}^{0}$ and $\tilde{\chi}_{1}^{0}$ is chosen to ensure this so that the largest possible signal rate can be estimated.

Note that the key difference between BP1 and BP2 lies in the choices of the lighter top squark and the LSP sneutrino mass. The relevant branching ratios of the $\tilde{\chi}_{2}^{0}$ and $\tilde{t}_{1}$ are similar for both of the benchmark points. $\operatorname{BR}\left(\tilde{\chi}_{1}^{ \pm} \rightarrow \ell \tilde{\nu}_{1,2}\right)$, where $\ell=e$ or $\mu$, is slightly different in the IH case due to the resultant $Y_{\nu}$ matrix with the comparatively bigger $Y_{\nu}^{11}$ required to fit the neutrino oscillation data subjected to the choice of $M^{11}$.

\section{B. Analysis and future reach}

We simulate the pair production of RH top squarks and its subsequent decays as already discussed for the two above-mentioned benchmark points. The events are generated at the parton level using MADGRAPH V2.5.5 [81,82] and subsequently passed through PYTHIA8 [83] for decay, showering, and hadronization. We use the parton distribution function set NN23LO1 [84,85]. Detector simulation is implemented via DELPHES V3.4.1 [86-88]. Jets are constructed at this stage using the anti- $k_{T}$ algorithm [89]. The ATLAS Collaboration recently included the same-sign trilepton channel in their search for top squarks in the context of the MSSM [28]. We implement the same analysis to obtain our signal rate. The top squark production cross section is appropriately scaled using the next-toleading-order (NLO) factor obtained from the LHC SUSY Cross Section Working Group webpage [90].

The electrons are primarily selected with $p_{T}>10 \mathrm{GeV}$ and $|\eta|<2.47$, excluding the region between the barrel and end-cap electromagnetic calorimeters, $1.37<|\eta|<1.52$. The muon candidates are selected with the same $p_{T}$ threshold and $|\eta|<2.5$. Jets are reconstructed with radius $R=0.4, p_{T}>20 \mathrm{GeV}$, and $|\eta|<2.8$. In our analysis we implement the $p_{T}$-dependent $b$-jet tagging efficiency and light jet misidentification efficiency following the ATLAS Collaboration criteria [28]. Finally, the $b$ jets are counted with $|\eta|<2.5$. The event selection further requires that at least two of the three same-sign leptons have $p_{T}>20 \mathrm{GeV}$. In order to reduce SM backgrounds arising from the mismeasurement of electron charge, events are vetoed if the invariant mass of two same-sign electrons is within a $10 \mathrm{GeV}$ window of the $Z$-boson mass. The ATLAS Collaboration obtained $1.6 \pm 0.8 \mathrm{SM}$ background events at the $13 \mathrm{TeV}$ LHC with these criteria in the $\ell^{ \pm} \ell^{ \pm} \ell^{ \pm}+\geq 1$ $b$-jet signal region with an integrated luminosity of $36 \mathrm{fb}^{-1}$ [28]. In Table II we show the signal cross sections corresponding to our benchmark points and the required luminosity to probe this scenario with $3 \sigma$ and $5 \sigma$ statistical significance $(\mathcal{S})$. In order to compute $\mathcal{S}$ of our signal $(S)$ over the SM background $(B)$ we use $\mathcal{S}=\frac{S}{\sqrt{B+\sigma_{B}^{2}}}$, where $\sigma_{B}$ is the uncertainty in the measurement of the SM background. For simplicity, even for the higher-luminosity estimates, we assume that $\sigma_{B}$ remains the same fraction 
TABLE I. Relevant parameters, masses, and branching ratios along with the relic density, direct detection cross section, and top squark pair production cross section at the $13 \mathrm{TeV}$ LHC corresponding to the two benchmark points for both $\mathrm{NH}$ and IH of light neutrino masses. $\mu_{S}^{i i}$ represents the three diagonal entries of the matrix $\mu_{S}$ and $M^{11}$ represents the first-generation diagonal entry in the matrix $M$. The other two diagonal entries $M^{22}$ and $M^{33}$ are kept fixed at $1000 \mathrm{GeV}$.

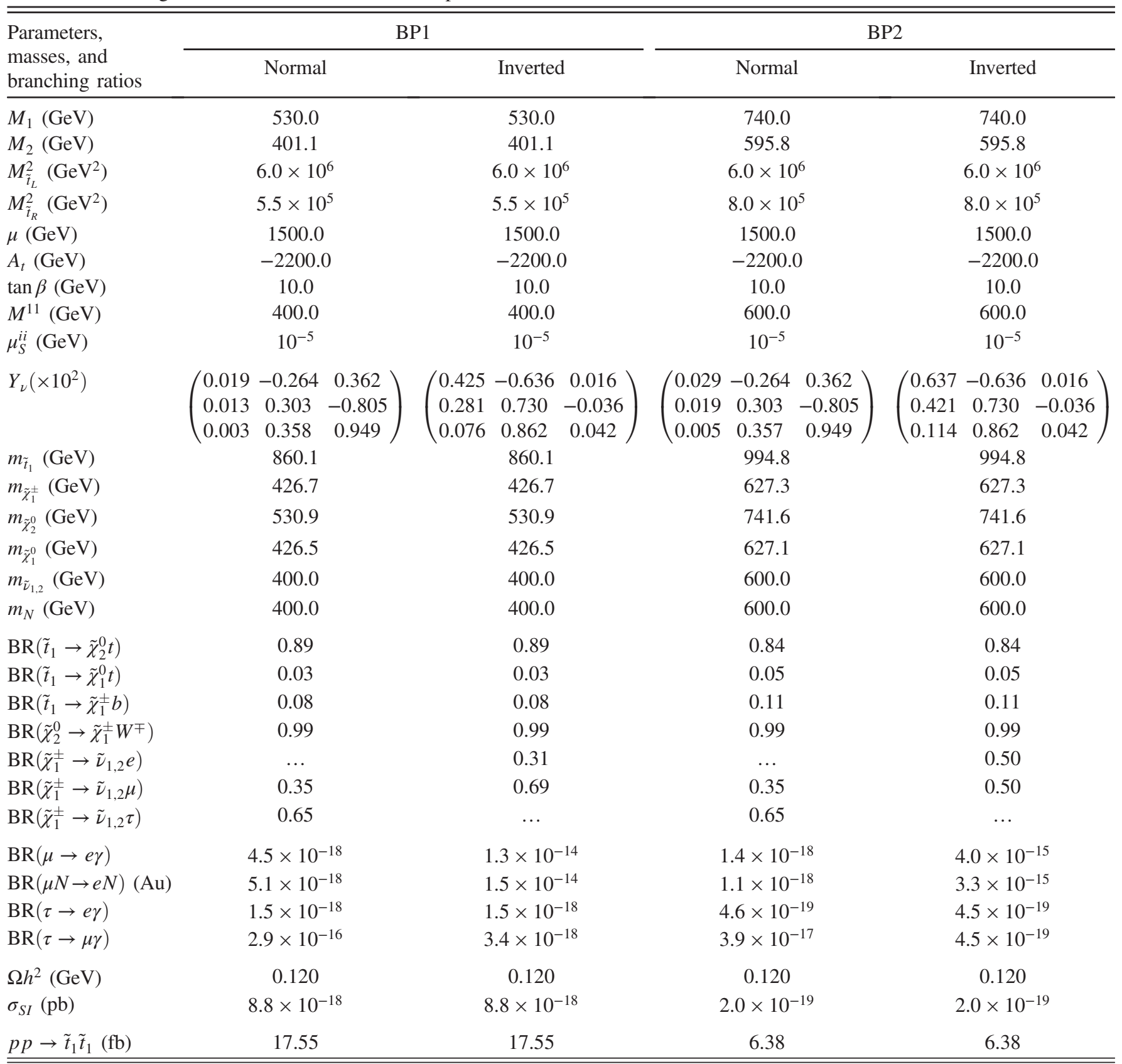

of $B$ as it is in $36 \mathrm{fb}^{-1}$ integrated luminosity. ${ }^{7}$ Note that the IH scenario for BP1 can already be ruled out from the accumulated data. However, the NH case needs an integrated luminosity $\sim 828.3 \mathrm{fb}^{-1}$ to achieve a $3 \sigma$ statistical significance. BP2, on the other hand, would require much higher luminosity owing to the larger top squark mass.

\footnotetext{
${ }^{7}$ We were forced to make this choice due to a lack of information, resulting in a conservative estimate.
}

The discovery significance in the NH case is beyond the reach of even $3000 \mathrm{fb}^{-1}$ integrated luminosity. However, for the IH case, $\mathcal{S}=3 \sigma$ can be achieved at $\sim 845.8 \mathrm{fb}^{-1}$.

As evident from Table II, the top squark mass probe in the present scenario depends heavily on the choice of neutrino mass hierarchy due to the variation in $\operatorname{BR}\left(\tilde{\chi}_{1}^{ \pm} \rightarrow\right.$ $\left.\ell \tilde{\nu}_{1,2}\right)$. To better understand the parameter space, we have used the existing LHC data to determine the exclusion limit on the lighter top squark mass subjected to $\mathrm{NH}$ and $\mathrm{IH}$ of 
TABLE II. Signal cross section and required integrated luminosity at the $13 \mathrm{TeV}$ LHC to probe the benchmark points with $3 \sigma$ and $5 \sigma$ statistical significance in the $\ell^{ \pm} \ell^{ \pm} \ell^{ \pm}+\geq 1 b$-jet signal region.

\begin{tabular}{lccccc}
\hline \hline & \multicolumn{2}{c}{ BP1 } & & \multicolumn{2}{c}{ BP2 } \\
\cline { 2 - 3 } \cline { 5 - 6 } Results & Normal & Inverted & & Normal & Inverted \\
\hline$\sigma_{\text {sig }}(\mathrm{fb})$ & 0.070 & 0.193 & & 0.026 & 0.070 \\
Required $\mathcal{L}\left(\mathrm{fb}^{-1}\right)(3 \sigma)$ & 828.3 & 12.2 & & $>3000$ & 845.8 \\
Required $\mathcal{L}\left(\mathrm{fb}^{-1}\right)(5 \sigma)$ & $>3000$ & 44.6 & & $>3000$ & $>3000$ \\
\hline \hline
\end{tabular}

light neutrino masses. For this, we keep the relative separation of the masses uniform throughout, i.e., $m_{\tilde{\chi}_{2}^{0}} \leq$ $m_{\tilde{t}_{1}}-m_{t}$ and $m_{h} \geq m_{\tilde{\chi}_{2}^{0}}-m_{\tilde{\chi}_{1}^{ \pm}}\left(m_{\tilde{\chi}_{1}^{0}}\right) \geq m_{W}$. The simple requirement of our signal region makes sure that the cut efficiency does not vary significantly for this spectrum choice over a wide range of top squark masses.
In Fig. 3 we show the chosen mass spectra in the framework of the SISM under consideration and the reach of top squark masses with existing data at the $13 \mathrm{TeV}$ LHC with $36 \mathrm{fb}^{-1}$ of data. The red horizontal line represents the 95\% confidence level exclusion limit on the visible cross section $\left(\sigma_{\mathrm{vis}}=0.11 \mathrm{fb}\right)$ in this signal region from the ATLAS Collaboration [28]. The violet and cyan lines represent the top squark mass reach in our model framework subjected to the existing data with $\mathrm{IH}$ and $\mathrm{NH}$ for the light neutrino masses, respectively, with the shaded regions already excluded. As evident, $m_{\tilde{t}_{1}}<815$ and $935 \mathrm{GeV}$ are already excluded under the assumptions of $\mathrm{NH}$ and $\mathrm{IH}$, respectively.

If some excess is found in this signal region at higher luminosity, it would be useful to have a prior idea of the mass range in which we can hope to discover the top squark with certainty. In order to determine this, we compute the discovery significance of the top squark lying within the
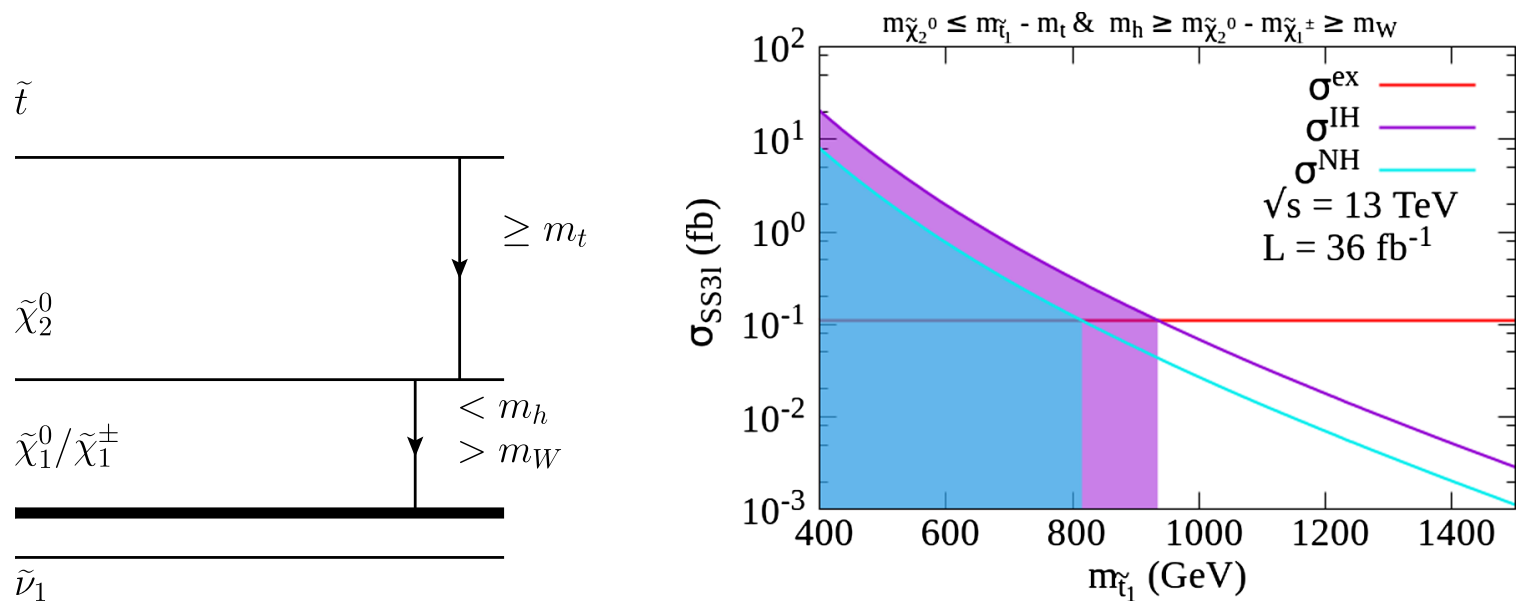

FIG. 3. Left: Choice of mass spectra. Right: The expected cross section in the same-sign trilepton final state vs the top squark mass and the exclusion obtained from the $36 \mathrm{fb}^{-1}$ luminosity data at the LHC. The red horizontal line corresponds to the experimental limit on the visible cross section [28].
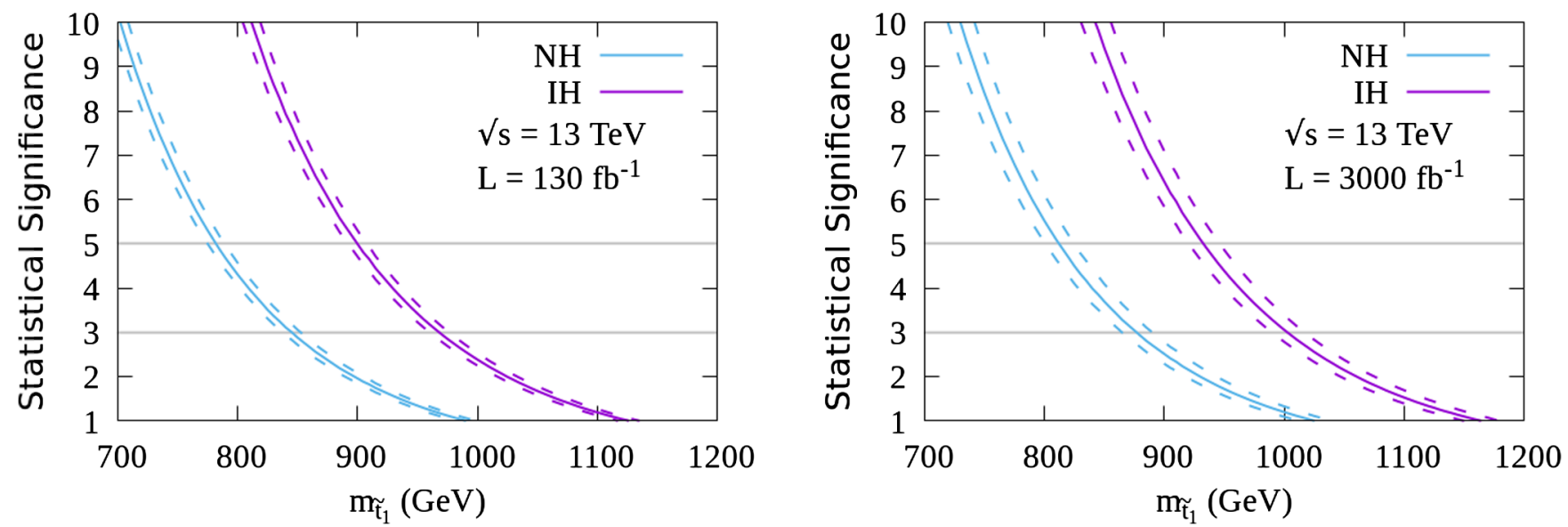

FIG. 4. Discovery reach in the top squark mass plane at the $\sqrt{s}=13 \mathrm{TeV}$ LHC with two choices for the integrated luminosity, $130 \mathrm{fb}^{-1}$ and $3000 \mathrm{fb}^{-1}$. The parallel grey lines indicate the $3 \sigma$ and $5 \sigma$ statistical significance requirements. 

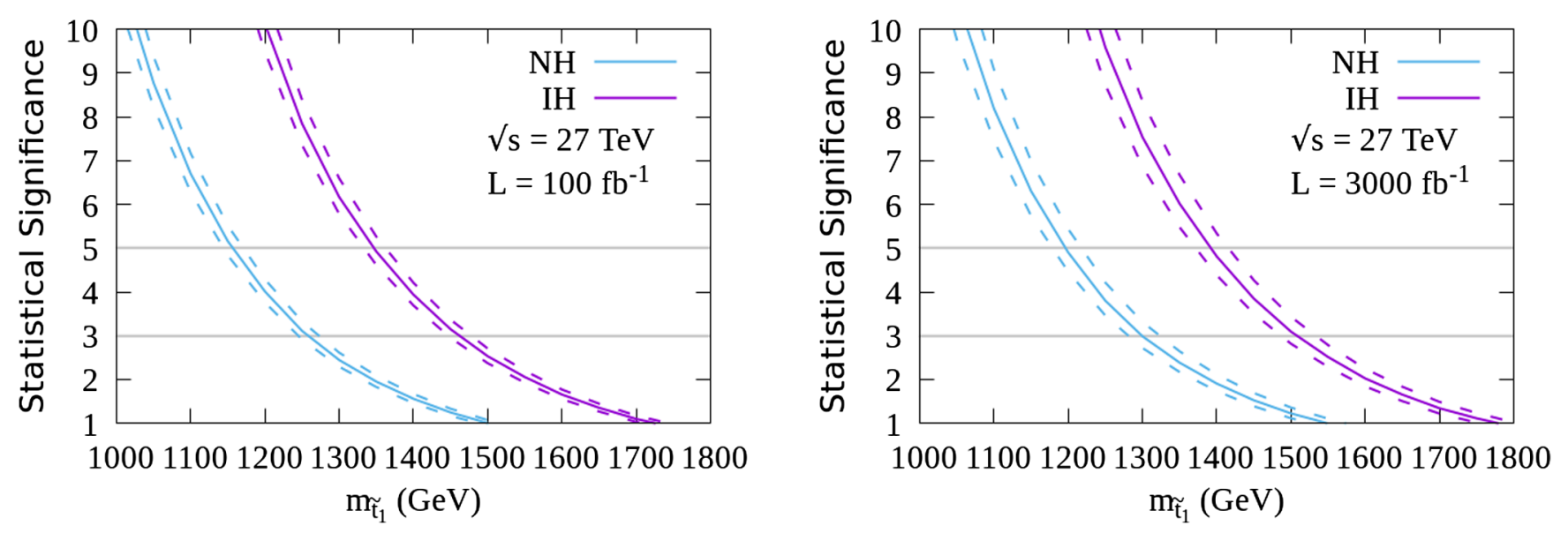

FIG. 5. Discovery reach in the top squark mass plane at a $\sqrt{s}=27 \mathrm{TeV}$ LHC with two choices for the integrated luminosity, $100 \mathrm{fb}^{-1}$ and $3000 \mathrm{fb}^{-1}$. The color coding is the same as in Fig. 4.

mass range $[700 \mathrm{GeV}-1.2 \mathrm{TeV}]$ at two different luminosities, $130 \mathrm{fb}^{-1}$ and $3000 \mathrm{fb}^{-1}$, with $\sqrt{s}=13 \mathrm{TeV}$. Figure 4 shows the distribution of the obtained statistical significance as a function of $m_{\tilde{t}_{1}}$. The horizontal grey lines in the figure represent the coveted $3 \sigma$ and $5 \sigma$ statistical significance requirements. The violet and cyan solid lines represent the prospects of our scenario subjected to the choice of IH or NH. The dotted lines around the solid ones are obtained by assuming a $10 \%$ variation in $\sigma_{B}$. As is evident from Fig. 4, at low luminosity $\left(130 \mathrm{fb}^{-1}\right)$ the discovery reach of $m_{\tilde{t}_{1}}$ can be up to $\sim 970 \mathrm{GeV}$ at most, whereas the highluminosity $\left(3000 \mathrm{fb}^{-1}\right)$ option can slightly improve the mass range up to $\sim 1 \mathrm{TeV}$. If IH in the neutrino masses is ruled out in the future by the neutrino oscillation experiments, the $m_{\tilde{t}_{1}}$ discovery reach cannot be beyond $900 \mathrm{GeV}$ even for high luminosities at the LHC.

Therefore, in spite of the small signal cross section, the top squark mass discovery reach can be in the $\mathrm{TeV}$ range in this signal region owing to the small SM background. The results do not improve drastically with the increase in the luminosity because of the presence of a large uncertainty factor in the SM background estimation. As more data is collected the statistical error is expected to decrease, which could significantly improve the top squark discovery reach. With a high center-of-mass-energy hadron collider becoming more and more relevant in the context of SUSY searches, we also estimate the projected discovery reach of the top squark mass in the present scenario at a $27 \mathrm{TeV}$ $p p$ collider. Our SM background computation at this center-of-mass energy from $t \bar{t} h, t \bar{t} W, t \bar{t} Z(\gamma), V V$, and $V V V$ production channels (where $V$ represents $W$ and $Z$ bosons) yields a cross section of $0.077 \mathrm{fb}$. We compute $\sigma_{B}$ following the same prescription as in Fig. 4. The NLO $k$-factor is computed with PROSPINO [91,92]. The $\sqrt{s}=$ $27 \mathrm{TeV}$ results are shown in Fig. 5. The increase in the signal cross section improves the discovery reach of $m_{\tilde{t}_{1}}$ to $\sim 1.55$ and $\sim 1.45 \mathrm{TeV}$ for $\mathrm{IH}$ and $\mathrm{NH}$, respectively.

\section{SUMMARY AND CONCLUSION}

In this work we have investigated the interplay between the neutrino mass mechanism, DM requirements, and top squark searches at the LHC in the context of the supersymmetric inverse seesaw scenario. The SISM scenario naturally gives rise to a right-sneutrino DM in the theory in the form of the LSP within an $R$-parity-conserving framework. Originating from a gauge singlet, the sneutrino must be close to the $125 \mathrm{GeV}$ Higgs resonance annihilation in order to produce the correct relic density. However, the present DM direct-detection constraints have put this parameter space in jeopardy, forcing us to look for the coannihilation of the right-sneutrino DM.

The sneutrino interactions are driven by the same Dirac neutrino Yukawa couplings introduced to fit the neutrino oscillation data. We have shown, using the Casas-Ibarra parameterization, that the lepton-flavor-violating constraints force these Yukawa parameters to be small. The choice of $y Y_{\nu}$ here is subjected to the choice of a heavy neutrino mass and the lepton-number-violating parameter $\mu_{S}$. Thus, the LFV constraints put a limit on the choices of $\mu_{S} \gtrsim 10^{-6} \mathrm{GeV}$ for sub-TeV heavy neutrino masses, in particular for IH. Among the constraints arising from the decay, $\mu \rightarrow e \gamma$ turns out to be the most severe. The predictions for $\tau \rightarrow \mu \gamma$ and $\tau \rightarrow \mu \mu \mu$ are relatively weaker, and constrain the sneutrino mass only in the $m_{\tilde{\nu}} \lesssim 100 \mathrm{GeV}$ range. The model predictions in the $\tau$ sector can be tested in future LFV searches. The constrained $Y_{\nu}$ from the LFV data results in a very small direct detection cross section of the sneutrino, which is beyond the present DM experimental sensitivity by a few orders of magnitude. This also forces the resonant contribution in the relic density to be small and motivates one to look for the coannihilation of the sneutrino DM with other sparticles. In this work, we have explored the sneutrino-wino coannihilation which has interesting implications for top squark searches at the LHC. 
The coannihilating DM scenario gives rise to a partially compressed electroweak spectra which makes the conventional top squark search strategies weaker. We have shown that the relic density is satisfied due to the coannihilation of the sneutrino with a wino-like neutralino and chargino, requiring the mass difference between the LSP and chargino/neutralino to be $\Delta m \sim 15-30 \mathrm{GeV}$ for all sneutrino mass values between $100-1000 \mathrm{GeV}$. The indirect limit on the LSP sneutrino mass arises from the wino mass bounds. However, the compression in the spectra again results in relatively weaker exclusions, allowing the LSP to be as light as $200 \mathrm{GeV}$ in this scenario.

Under such circumstances, conventional top squark search strategies prove to be ineffective in probing $\mathrm{TeV}$ scale top squark masses. Here we have studied top squark pair production and its subsequent decays into a top quark and a bino-like next-to-NLSP $\chi^{0}{ }_{2}$. The further decay of the bino into a gauge boson and a wino-like chargino $\tilde{\chi}^{ \pm}$and $\tilde{\chi}^{ \pm} \rightarrow \ell \tilde{\nu}_{1,2}$ leads to a novel same-sign trilepton signal $\ell^{ \pm} \ell^{ \pm} \ell^{ \pm}+\geq 1 b-$ jets $+E_{T}$ for the top squark at the LHC. Unlike the conventional top squark search channels, this signal region has very small SM background. As a result, despite the small signal rate, the top squark mass can be probed close to 935 (815) $\mathrm{GeV}$ with the existing LHC data for $\mathrm{IH}(\mathrm{NH})$ of neutrino masses. Stop mass limits prove to be stronger for the inverted hierarchy in the light neutrino masses due to the greater leptonic branching ratio of the chargino, resulting in a greater abundance of electrons in the final state, which also serves as a distinguishing feature of this model from the MSSM. In the MSSM with a neutralino LSP, however, this same-sign trilepton signal rate will be much lower. A $3 \sigma$ discovery significance can be obtained with $m_{\tilde{t}_{1}} \sim 1 \mathrm{TeV}$ at high luminosity with a $13 \mathrm{TeV}$ center-of-mass energy. We have also shown that the top squark mass discovery reach can go beyond $1.5 \mathrm{TeV}$ at a high-energy $p p$ collider with a $27 \mathrm{TeV}$ center-of-mass energy. With improved sensitivity in SM background measurements at higher luminosity, the conservative limits we obtained are bound to improve significantly.

\section{ACKNOWLEDGMENTS}

S. M. and K. H. acknowledge H2020-MSCA-RICE2014 Grant No. 645722 (NonMinimal Higgs). S. M. would like to thank IACS, Kolkata for hospitality during the final phase of this work. M. M. would like to acknowledge the DST-INSPIRE research Grant No. IFA14-PH-99.
[1] M. C. Gonzalez-Garcia, M. Maltoni, and T. Schwetz, Nucl. Phys. B908, 199 (2016).

[2] P. F. de Salas, D. V. Forero, C. A. Ternes, M. Tortola, and J. W. F. Valle, Phys. Lett. B 782, 633 (2018).

[3] P. Minkowski, Phys. Lett. 67B, 421 (1977).

[4] T. Yanagida, in Proceedings of the Workshop on the Baryon Number of the Universe and Unified Theories, Tsukuba, Japan, 1979.

[5] R. N. Mohapatra and G. Senjanovic, Phys. Rev. Lett. 44, 912 (1980).

[6] S. L. Glashow, NATO Adv. Study Inst. Ser. B Phys. 59, 687 (1980).

[7] M. Gell-Mann, P. Ramond, and R. Slansky, (CERN).

[8] R. N. Mohapatra, Phys. Rev. Lett. 56, 561 (1986).

[9] R. N. Mohapatra and J. W. F. Valle, Phys. Rev. D 34, 1642 (1986).

[10] M. C. Gonzalez-Garcia and J. W. F. Valle, Phys. Lett. B 216 , 360 (1989).

[11] A. Das and N. Okada, Phys. Rev. D 88, 113001 (2013).

[12] A. Das and N. Okada, Phys. Rev. D 93, 033003 (2016).

[13] A. Das and N. Okada, Phys. Lett. B 774, 32 (2017).

[14] H.-S. Lee, K. T. Matchev, and S. Nasri, Phys. Rev. D 76, 041302 (2007).

[15] D. G. Cerdeno, C. Munoz, and O. Seto, Phys. Rev. D 79, 023510 (2009).

[16] S. Mondal, S. Biswas, P. Ghosh, and S. Roy, J. High Energy Phys. 05 (2012) 134.
[17] P. S. B. Dev, S. Mondal, B. Mukhopadhyaya, and S. Roy, J. High Energy Phys. 09 (2012) 110.

[18] V. De Romeri and M. Hirsch, J. High Energy Phys. 12 (2012) 106.

[19] S. Banerjee, P. S. B. Dev, S. Mondal, B. Mukhopadhyaya, and S. Roy, J. High Energy Phys. 10 (2013) 221.

[20] D. K. Ghosh, S. Mondal, and I. Saha, J. Cosmol. Astropart. Phys. 02 (2015) 035.

[21] J. Chang, K. Cheung, H. Ishida, C.-T. Lu, M. Spinrath, and Y.-L. S. Tsai, J. High Energy Phys. 10 (2017) 039.

[22] J. Chang, K. Cheung, H. Ishida, C.-T. Lu, M. Spinrath, and Y.-L.S. Tsai, J. High Energy Phys. 09 (2018) 071.

[23] L. Delle Rose, S. Khalil, S. J. D. King, C. Marzo, S. Moretti, and C. S. Un, Phys. Rev. D 96, 055004 (2017).

[24] L. Delle Rose, S. Khalil, S. J. D. King, S. Kulkarni, C. Marzo, S. Moretti, and C. S. Un, J. High Energy Phys. 07 (2018) 100.

[25] L. Delle Rose, S. Khalil, S. J. D. King, S. Kulkarni, C. Marzo, S. Moretti, and C. S. Un, arXiv:1804.09470.

[26] T. Falk, K. A. Olive, and M. Srednicki, Phys. Lett. B 339, 248 (1994).

[27] T. Hebbeker, Phys. Lett. B 470, 259 (1999).

[28] M. Aaboud et al. (ATLAS Collaboration), J. High Energy Phys. 09 (2017) 084.

[29] M. Aaboud et al. (ATLAS Collaboration), J. High Energy Phys. 08 (2017) 006. 
[30] M. Aaboud et al. (ATLAS Collaboration), Eur. Phys. J. C 77, 898 (2017).

[31] M. Aaboud et al. (ATLAS Collaboration), J. High Energy Phys. 11 (2017) 195.

[32] M. Aaboud et al. (ATLAS Collaboration), J. High Energy Phys. 12 (2017) 085.

[33] M. Aaboud et al. (ATLAS Collaboration), J. High Energy Phys. 06 (2018) 108.

[34] M. Aaboud et al. (ATLAS Collaboration), Phys. Rev. D 98, 032008 (2018).

[35] F. F. Deppisch, P. S. B. Dev, and A. Pilaftsis, New J. Phys. 17, 075019 (2015).

[36] S. Khalil, Phys. Rev. D 82, 077702 (2010).

[37] P. S. B. Dev and R. N. Mohapatra, Phys. Rev. D 81, 013001 (2010).

[38] F. Deppisch and J. W. F. Valle, Phys. Rev. D 72, 036001 (2005).

[39] M. Hirsch, T. Kernreiter, J. C. Romao, and A. V. del Moral, J. High Energy Phys. 01 (2010) 103.

[40] A. Abada, D. Das, A. Vicente, and C. Weiland, J. High Energy Phys. 09 (2012) 015.

[41] A. Abada, M. E. Krauss, W. Porod, F. Staub, A. Vicente, and C. Weiland, J. High Energy Phys. 11 (2014) 048.

[42] J. A. Casas and A. Ibarra, Nucl. Phys. B618, 171 (2001).

[43] K.-S. Sun, T.-F. Feng, G.-H. Luo, X.-Y. Yang, and J.-B. Chen, Mod. Phys. Lett. A 28, 1350151 (2013).

[44] X. Marcano, Lepton flavor violation from low scale seesaw neutrinos with masses reachable at the LHC, Ph.D. thesis, Madrid, Autonoma University, 2017.

[45] L. Lello and D. Boyanovsky, Phys. Rev. D 87, 073017 (2013).

[46] A. Blondel, E. Graverini, N. Serra, and M. Shaposhnikov (FCC-ee study Team), Proceedings of the 37th International Conference on High Energy Physics (ICHEP 2014): Valencia, Spain, 2014, Nucl. Part. Phys. Proc. 273-275, 1883 (2016).

[47] M. Anelli et al. (SHiP Collaboration), arXiv:1504.04956.

[48] A. Abada, D. Das, and C. Weiland, J. High Energy Phys. 03 (2012) 100.

[49] J. Adam et al. (MEG Collaboration), Phys. Rev. Lett. 110, 201801 (2013).

[50] A. M. Baldini et al., arXiv:1301.7225.

[51] B. Aubert et al. (BABAR Collaboration), Phys. Rev. Lett. 104, 021802 (2010).

[52] T. Aushev et al., arXiv:1002.5012.

[53] K. Hayasaka et al., Phys. Lett. B 687, 139 (2010).

[54] W. H. Bertl et al. (SINDRUM II Collaboration), Eur. Phys. J. C 47, 337 (2006).

[55] L. Bartoszek et al. (Mu2e Collaboration), arXiv:1501.05241.

[56] W. Porod, Comput. Phys. Commun. 153, 275 (2003).

[57] W. Porod and F. Staub, Comput. Phys. Commun. 183, 2458 (2012).

[58] W. Porod, J. High Energy Phys. 05 (2002) 030.

[59] F. Staub, arXiv:0806.0538.

[60] F. Staub, Comput. Phys. Commun. 181, 1077 (2010).

[61] F. Staub, Comput. Phys. Commun. 182, 808 (2011).

[62] F. Staub, Comput. Phys. Commun. 185, 1773 (2014).

[63] F. Staub, Adv. High Energy Phys. 2015, 840780 (2015).
[64] P. Adamson et al. (NOvA Collaboration), Phys. Rev. Lett. 118, 231801 (2017).

[65] M. A. Acero et al. (NOvA Collaboration), Phys. Rev. D 98 , 032012 (2018).

[66] G. Hinshaw et al. (WMAP Collaboration), Astrophys. J. Suppl. Ser. 208, 19 (2013).

[67] E. Aprile et al. (XENON Collaboration), Phys. Rev. Lett. 119, 181301 (2017).

[68] X. Cui et al. (PandaX-II Collaboration), Phys. Rev. Lett. 119, 181302 (2017).

[69] E. Aprile et al. (XENON Collaboration), Phys. Rev. Lett. 121, 111302 (2018).

[70] C. Arina and M. E. Cabrera, J. High Energy Phys. 04 (2014) 100.

[71] G. Belanger, F. Boudjema, A. Pukhov, and A. Semenov, Comput. Phys. Commun. 185, 960 (2014).

[72] M. Aaboud et al. (ATLAS Collaboration), Eur. Phys. J. C 78, 995 (2018).

[73] A. M. Sirunyan et al. (CMS Collaboration), J. High Energy Phys. 03 (2018) 160.

[74] M. Aaboud et al. (ATLAS Collaboration), Phys. Rev. D 97, 052010 (2018).

[75] M. Aaboud et al. (ATLAS Collaboration), J. High Energy Phys. 06 (2018) 022.

[76] A. M. Sirunyan et al. (CMS Collaboration), J. High Energy Phys. 03 (2018) 166.

[77] M. Aaboud et al. (ATLAS Collaboration), Eur. Phys. J. C 78, 154 (2018).

[78] A. M. Sirunyan et al. (CMS Collaboration), J. High Energy Phys. 11 (2017) 029.

[79] M. Drees, H. Dreiner, D. Schmeier, J. Tattersall, and J. S. Kim, Comput. Phys. Commun. 187, 227 (2015).

[80] D. Dercks, N. Desai, J. S. Kim, K. Rolbiecki, J. Tattersall, and T. Weber, Comput. Phys. Commun. 221, 383 (2017).

[81] J. Alwall, M. Herquet, F. Maltoni, O. Mattelaer, and T. Stelzer, J. High Energy Phys. 06 (2011) 128.

[82] J. Alwall, R. Frederix, S. Frixione, V. Hirschi, F. Maltoni, O. Mattelaer, H. S. Shao, T. Stelzer, P. Torrielli, and M. Zaro, J. High Energy Phys. 07 (2014) 079.

[83] T. Sjöstrand, S. Ask, J. R. Christiansen, R. Corke, N. Desai, P. Ilten, S. Mrenna, S. Prestel, C. O. Rasmussen, and P.Z. Skands, Comput. Phys. Commun. 191, 159 (2015).

[84] R. D. Ball et al., Nucl. Phys. B867, 244 (2013).

[85] R. D. Ball et al. (NNPDF Collaboration), J. High Energy Phys. 04 (2015) 040.

[86] J. de Favereau, C. Delaere, P. Demin, A. Giammanco, V. Lemaître, A. Mertens, and M. Selvaggi (DELPHES 3 Collaboration), J. High Energy Phys. 02 (2014) 057.

[87] M. Selvaggi, J. Phys. Conf. Ser. 523, 012033 (2014).

[88] A. Mertens, J. Phys. Conf. Ser. 608, 012045 (2015).

[89] M. Cacciari, G. P. Salam, and G. Soyez, J. High Energy Phys. 04 (2008) 063.

[90] https://twiki.cern.ch/twiki/bin/view/LHCPhysics/ SUSYCrossSections.

[91] W. Beenakker, R. Hopker, and M. Spira, arXiv:hep-ph/ 9611232.

[92] W. Beenakker, M. Kramer, T. Plehn, M. Spira, and P. M. Zerwas, Nucl. Phys. B515, 3 (1998). 